\title{
Diet-quality and its association with cardiovascular diseases and cancer incidence and all-cause mortality: a prospective cohort study from UK Biobank
}

\author{
Fanny Petermann-Rocha ${ }^{1,2}$, Stuart R. Gray ${ }^{2}$ Jill Pell $^{1}$ and Carlos Celis-Morales ${ }^{2}$ \\ ${ }^{1}$ Institute of Health and Wellbeing, University of Glasgow, Glasgow, United Kingdom and \\ ${ }^{2}$ BHF Glasgow Cardiovascular Research Centre, Institute of Cardiovascular and Medical Science, University of \\ Glasgow, Glasgow, United Kingdom
}

\begin{abstract}
Introduction- Newly available data from big scale studies conducted in the UK, such as the UK Biobank, offers the possibility to further explore the prospective association between a diet-quality score and health outcomes after accounting for the effect of important confounding factors. The aim of this work, therefore, was to investigate the association between a diet-quality score, with the incidence of cardiovascular diseases (CVDs), cancer and all-cause mortality.

Material and methods- This study includes 345,343 participants (age range: 39-73, 55.1\% women) from the UK Biobank, a prospective population-based study. Using 21 standardised variables of diet (alcohol, bread, bread type, cereal, dried fruit, water, coffee, tea, cheese, oily fish, non-oily fish, salt added to food, spread type, fresh fruit, cooked vegetable, raw vegetables, milk type, poultry, beef, lamb, and pork) we created a diet-quality score (very healthy, healthy, unhealthy and very unhealthy) using principal-component factor analysis. Associations between the dietary-quality score (very unhealthy individuals were the reference group) and health outcomes (all-cause mortality, CVD and cancer incidence) were investigated using Cox-proportional hazard models. All analyses were performed using STATA 14 statistical software.

Results- In comparison to individuals with a very unhealthy diet, those with a better diet-quality had a lower risk of all-cause mortality and cancer as well as incidence of CVD and cancer. For example, individuals classified in the very healthy group had a $12 \%$ lower risk of all-cause mortality (HR: 0.88 [95\% CI: 0.82 to 0.95$]$ ), $12 \%$ lower risk of CVD incidence (HR: 0.88 [95\% CI: 0.80 to 0.98 ]), $17 \%$ of all-cancer mortality (HR: 0.83 [95\% CI: 0.75 to 0.93 ]), and 10\% lower risk all-cancer incidence (HR: 0.90 [95\% CI: 0.85 to 0.94 ]). Those in the healthy group had a 12\% lower risk of all-cause (HR: 0.88 [95\% CI: 0.83 to 0.93 ]) and $15 \%$ lower risk of all-cancer mortality (HR: 0.85 [95\% CI: 0.78 to 0.93 ]). There was no significant association between CVD mortality and any diet-quality group. These findings were independent of major confounding factors including socio-demographic covariates, prevalent of diseases and lifestyle factors.
\end{abstract}

Discussion- Our findings indicate that individuals with a healthy diet in the UK biobank cohort are associated with a lower risk of premature mortality, and incidence of CVDs and cancer independently of major confounding factors.

\section{Conflict of Interest}

There is no conflict of interest 